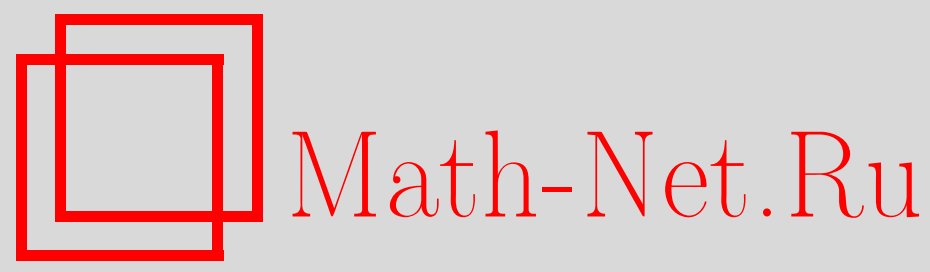

И. К. Дассиос, О решениях и алгебраической двойственности обобщенных линейных систем с дискретным временем, Дискрет. матем., 2012, том 24, выпуск 4, 131-146

DOI: https://doi.org/10.4213/dm1216

Использование Общероссийского математического портала Math-Net.Ru подразумевает, что вы прочитали и согласны с пользовательским соглашением http://www.mathnet.ru/rus/agreement

Параметры загрузки:

IP: 3.80 .181 .102

26 апреля 2023 г., 14:03:57 


\title{
О решениях и алгебраической двойственности обобщенных линейных систем с дискретным временем
}

() 2012 г. И. К. Дассиос

\begin{abstract}
В статье изучается класс обобщенных линейных систем с дискретным временем, коэффициентами которых являются квадратные матрицы, состоящие из констант. Целью статьи является установление связи между решениями некоторого класса сингулярных линейных систем с дискретным временем с их собственными и транспонированными двойственными системами. Вначале мы исследуем первичную систему и с использованием инвариантов ее матричного пучка получаем необходимые и достаточные условия для существования и единственности решения и выводим формулы для решений. Затем мы показываем, что с использованием того же матричного пучка можно изучать существование и единственность решения для собственной и транспонированной двойственной системы. Более того, их решения, если они существуют, могут быть для них явно выписаны без дальнейших вычислений. В заключение в иллюстративных целях мы приводим численный пример.
\end{abstract}

\section{1. Введение}

Под линейными системами с дискретным временем мы будем подразумевать системы, в которых переменные принимают значения в отдельно взятые моменты времени. Системы с дискретным временем отличаются от таковых с непрерывным временем в том, что их сигналы предстают в виде выборок. С развитием цифровых вычислительных машин, теория систем с дискретным временем начинает играть ведущую роль в теории управления. На практике, системы с дискретным временем возникают в результате взятия выборок из систем с непрерывным временем или в случае, когда возможно использовать только дискретные данные. Системы с дискретным временем находят широкое применение в экономике, физике, теории схем и других областях. Например, в финансовой математике хорошо известна модель Леонтьева [1], или модель роста населения Лесли, см. также [1]. В медицине известны модели хозяина-паразита, см. [2]. Исследования поглощающих марковских цепей, а также распределения тепла через теплопроводящий стержень также являются интересными применениями, предложенными в [2]. Вообще, имеется достаточно большое число исследований, посвященных системам с дискретным временем, см. $[1-5,7-11,13,15-20]$. Обобщенная автономная система с дискретным временем вида

$$
F Y_{k+1}=G Y_{k}
$$


с заданными начальными условиями

$$
Y_{k_{0}}
$$

возникает как единое представление при изучении свойств линейных систем, где $F, G \in$ $M(n \times n ; \mathscr{F}), Y_{k} \in \mathcal{M}(n \times 1 ; \mathscr{F})$, иными словами, взяты из алгебры матриц с элементами из поля $\mathscr{F}$, в то время как матрица $F$ вырождена. Для краткости положим

$$
M_{n}=\mathcal{M}(n \times n ; \mathscr{F}), \quad M_{n m}=\mathcal{M}(n \times m ; \mathscr{F}) .
$$

Важность систем вида (1) также связана с их ролью в определении ряда ключевых понятий двойственности, см. [2]. Понятия двойственной конфигурации и двойственной задачи ведут происхождение из проективной геометрии, см. [5]. Сущность понятия двойственной системы, определяемой ниже, подобна используемому в проективной геометрии для автономных дифференциальных систем. Использование этого принципа подразумевает, что если некоторое предложение верно для заданной системы, то двойственное предложение верно для двойственной системы. Рассмотрим систему, определяемую как

$$
F \widehat{Y}_{k}=G \widehat{Y}_{k+1}
$$

с заданными начальными условиями

$$
\widehat{Y}_{k_{0}}
$$

и систему, определяемую как

$$
F^{T} \tilde{Y}_{k+1}=G^{T} \tilde{Y}_{k}
$$

с заданными начальными условиями

$$
\tilde{Y}_{k_{0}}
$$

где $(\cdot)^{T}$ означает транспонирование.

Определение 1. Систему (1) назовем первичной системой, систему (3) назовем собственной двойственной, или просто двойственной, и систему (5) назовем транспонированной двойственной.

Двойственность между системами (1) и (3) ранее получила название дифференциалинтегральной двойственности, а двойственность между (1) и (5) - двойственности транспонирования. Для изучения линейных матричных дифференциальных уравнений (ЛМДУ) с неизменными во времени коэффициентами широко используется теория матричных пучков, см., например, [1-6, 8, 10-20]. Под матричным пучком понимается семейство матриц $s F-G$, параметризованное комплексным числом $s$ (см. $[7,8])$. Если $G$ квадратна и $F=I_{n}$, где $I_{n}-$ единичная матрица, то нули функции $\operatorname{det}(s F-G)$ являются собственными значениями $G$. Таким образом, проблему нахождения нетривиального решения уравнения

$$
s F X=G X
$$

естественно назвать обобщенной проблемой собственных значений. Хотя эта задача и выглядит как простое обобщение обычной проблемы собственных значений, имеется ряд важных различий.

Во-первых, $\operatorname{det}(s F-G)$ может быть тождественным нулем, вне зависимости от $s$. 
Во-вторых, $F$ может быть вырожденной, и тогда возникают бесконечные собственные значения. Чтобы увидеть это, запишем обобщенную проблему собственных значений в обратной форме:

$$
F X=s^{-1} G X
$$

Если $F$ вырождена с нулевым вектором $X$, то $G X=0_{n, 1}$, так что $X$ является собственным вектором обратной проблемы, соответствующим собственному значению $s^{-1}=0$, то есть $s=\infty$.

Определение 2. При известных $F, G \in M_{n m}$ и неопределенном $s \in F$, матричный пучок $s F-G$ называется регулярным, если $m=n$ и $\operatorname{det}(s F-G) \neq 0$. В противном случае пучок называется сингулярным.

В настоящей статье мы рассматриваем случай, когда пучок регулярен. Для системы (1) соответствующий матричный пучок есть $s F-G$, для системы (3) матричный пучок есть $F-\hat{s} G$, и для системы (5) он есть $\tilde{s} F^{T}-G^{T}$. Ясно, что вышеописанная двойственность по сути определяется связями между соответствующими пучками. Изучение двойственностей между (1), (3) и (5) сводится к изучению связей между их пучками. Понятие двойственности, введенное выше, может быть интерпретировано алгебраически, через соотношения между инвариантами строгой эквивалентности соответствующих пучков. Основной результат заключается в том, что если известно решение одной из этих систем, то решения остальных двух систем легко получить без дальнейших громоздких вычислений.

\section{2. Регулярные матричные пучки}

В настоящей статье мы рассматриваем исключительно регулярные матричные пучки.

Линейные системы вида (1) с заданными начальными условиями (2) тесно связаны с теорией матричных пучков, поскольку их алгебраические, геометрические и динамические свойства определяются структурой соответствующего пучка $s F-G$. Класс $s F-G$ характеризуется однозначно определенным элементом, носящим имя комплексной канонической формы Вейерштрасса $s F_{w}-Q_{w}$ (см. $\left.[5,6,9]\right)$ задаваемой полным множеством инвариантов $s F-G$. В случае регулярности $s F-G$, получаем собственные значения следующих типов:

- нулевые собственные значения алгебраической кратности $p_{0}$;

- ненулевые конечные собственные значения $a_{i}$ алгебраической кратности $p_{i}$;

- бесконечные собственные значения алгебраической кратности $q$.

Предположим, что

$$
\sum_{i=1}^{v} p_{i}=p, \quad p_{0}+p+q=n .
$$

Определение 3. Пусть $B_{1}, B_{2}, \ldots, B_{n}$ есть элементы $M_{n}$. Их прямая сумма, обозначаемая $B_{1} \oplus B_{2} \oplus \ldots \oplus B_{n}$, есть blockdiag $\left[\begin{array}{llll}B_{1} & B_{2} & \ldots & B_{n}\end{array}\right]$. 
В силу регулярности $s F-G$, найдутся невырожденные матрицы $P, Q \in \mathcal{M}_{n}$, такие, что

$$
\begin{aligned}
& P F Q=F_{w}=I_{p_{0}} \oplus I_{p} \oplus H_{q}, \\
& P G Q=G_{w}=J_{p_{0}} \oplus J_{p} \oplus I_{q},
\end{aligned}
$$

где $s F_{w}-Q_{w}$ есть комплексная форма Вейерштрасса регулярного пучка $s F-G$, определенная как

$$
s F_{w}-Q_{w}=s I_{p_{0}}-J_{p_{0}} \oplus s I_{p}-J_{p} \oplus s H_{q}-I_{q},
$$

где первые два нормальных жорданова элемента $s I_{p_{0}}-J_{p_{0}}$ и $s I_{p}-J_{p}$ однозначно определяются множеством конечных собственных значений $s F-G$, нулевых и ненулевых соответственно:

$$
s^{p_{0}}\left(s-a_{1}\right)^{p_{1}}, \ldots,\left(s-a_{v}\right)^{p_{v}} .
$$

Второй блок имеет вид

$$
s I_{p}-J_{p}=s I_{p_{1}}-J_{p_{1}}\left(a_{1}\right) \oplus \ldots \oplus s I_{p_{v}}-J_{p_{v}}\left(a_{v}\right) .
$$

Блоки третьего типа количеством $q$ штук однозначно определяют блок $s H_{q}-I_{q}$, соответствующий бесконечным собственным значениям $s F-G$

$$
\hat{s}^{q_{1}}, \ldots, \hat{s}^{q_{\sigma}}, \quad \sum_{j=1}^{\sigma} q_{j}=q,
$$

и имеющий вид

$$
s H_{q}-I_{q}=s H_{q_{1}}-I_{q_{1}} \oplus \ldots \oplus s H_{q_{\sigma}}-I_{q_{\sigma}} .
$$

Таким образом, $H_{q}$ есть нильпотентный элемент $M_{n}$ с индексом

$$
\tilde{q}=\max \left\{q_{j}: j=1,2, \ldots, \sigma\right\},
$$

где

$$
H_{q}^{\tilde{q}}=0_{q, q},
$$

и $I_{p_{j}}, J_{p_{j}}\left(a_{j}\right), H_{q_{j}}$ определены следующим образом:

$$
\begin{aligned}
& I_{p_{j}}=\left[\begin{array}{ccccc}
1 & 0 & \ldots & 0 & 0 \\
0 & 1 & \ldots & 0 & 0 \\
\ldots & \ldots & \ldots & \ldots \\
0 & 0 & \ldots & 0 & 1
\end{array}\right] \in \mathcal{M}_{p_{j}} \\
& J_{p_{j}}\left(a_{j}\right)=\left[\begin{array}{ccccc}
a_{j} & 1 & \ldots & 0 & 0 \\
0 & a_{j} & \ldots & 0 & 0 \\
\ldots & \ldots & \ldots & \ldots & \ldots \\
0 & 0 & \ldots & a_{j} & 1 \\
0 & 0 & \ldots & 0 & a_{j}
\end{array}\right] \in M_{p_{j}} \\
& H_{q_{j}}=\left[\begin{array}{ccccc}
0 & 1 & \ldots & 0 & 0 \\
0 & 0 & \ldots & 0 & 0 \\
\ldots & \ldots \ldots & \ldots & \ldots \\
0 & 0 & \ldots & 0 & 1 \\
0 & 0 & \ldots & 0 & 0
\end{array}\right] \in M_{q_{j}}
\end{aligned}
$$

Сведения об алгоритмах, относящихся к жордановым матрицам, можно найти в $[5,7,19]$. 
Замечание 1. Легко видеть, что

$$
J_{p_{0}}=H_{p_{0}} .
$$

Матрица $Q$ конструируется из $n$ (обобщенных) линейно независимых собственных векторов, соответствующих нулевым, ненулевым и бесконечным собственным значениям.

Двойственность между (1), (3) или между их матричными пучками $s F-G, F-\hat{s} G$ может рассматриваться как следствие специального билинейного или проективного преобразования на прямой:

$$
s \longrightarrow \frac{1}{\hat{s}}
$$

которое, очевидно, преобразует точки $0, a_{i} \neq 0, \infty$ на компактифицированной комплексной плоскости в точки $\infty, 1 / a_{i}, 0$ соответственно. Понятие двойственности может пониматься алгебраически, в терминах отношений между инвариантами строгой эквивалентности соответствующих пучков. Эти отношения таковы:

- нулевое собственное значение $s F-G$ есть бесконечное собственное значение $F-\hat{s} G$ и обратно;

- ненулевое конечное собственное значение $a_{i}$ определяет ненулевое конечное собственное значение $1 / a_{i} F-\hat{s} G$ и обратно;

- бесконечное собственное значение $s F-G$ есть нулевое собственное значение $F-\hat{s} G$ и обратно.

Очевидно, основной характеристикой этого вида двойственности является инверсия, определяемая двойственной ролью различных типов собственных значений.

\section{3. Основные результаты}

В этом разделе мы получим формулы для решений ЛМдУ с регулярным матричным пучком и дадим необходимые и достаточные условия для существования и единственности решений.

Для регулярного матричного пучка системы (1) найдутся невырожденные матрицы $P, Q \in M_{n}$, такие, что

$$
\begin{aligned}
& P F Q=F_{w}=I_{p_{0}} \oplus I_{p} \oplus H_{q}, \\
& P G Q=G_{w}=J_{p_{0}} \oplus J_{p} \oplus I_{q} .
\end{aligned}
$$

Положим

$$
P=\left[\begin{array}{c}
P_{p_{0}} \\
P_{p} \\
P_{q}
\end{array}\right], \quad P_{p_{0}} \in M_{p_{0} n}, \quad P_{p} \in \mathcal{M}_{p n}, \quad P_{p} \in M_{p n},
$$

и

$$
Q=\left[\begin{array}{lll}
Q_{p_{0}} & Q_{p} & Q_{q}
\end{array}\right],
$$

где $Q_{p_{0}} \in M_{n p_{0}}$ - матрица, столбцы которой есть $p_{0}$ линейно независимых (обобщенных) собственных векторов, соответствующих $p_{0}$ нулевым собственным значениям $s F-G$, 
$Q_{p} \in M_{(n) p}-$ матрица, столбцы которой есть $p$ линейно независимых (обобщенных) собственных векторов, соответствующих $p$ конечным (нулевым и ненулевым) собственным значениям $s F-G$, и $Q_{q} \in \mathcal{M}_{(m n) q}$ - матрица, столбцы которой есть $q$ линейно независимых (обобщенных) собственных векторов, соответствующих $q$ бесконечным собственным значениям $s F-G$ или $q$ нулевым собственным значениям $F-\hat{s} G$.

Теорема 1. Рассмотрим системы (1), (3), (5) с заданными начальными условиями (2), (4), (6), соответственно, и пусть $P, Q$-невырожденные матрицы, определенные (14). Тогда

(1) решение системы (1) единственно тогда и только тогда, когда

$$
Y_{k_{0}} \in \operatorname{colspan}\left[Q_{p_{0}} \quad Q_{p}\right] \text {; }
$$

ее единственное решение дается соотношением

$$
Y_{k}=\left[\begin{array}{ll}
Q_{p_{0}} & Q_{p}
\end{array}\right] J_{p_{0}+p}^{k-k_{0}} Z_{k_{0}}^{p_{0}+p},
$$

где

$$
J_{p_{0}+p}^{k-k_{0}}=J_{p_{0}}^{k-k_{0}} \oplus J_{p}^{k-k_{0}}
$$

и $Z_{k_{0}}^{p_{0}+p}$ есть единственное решение алгебраической системь

$$
Y_{k_{0}}=\left[\begin{array}{ll}
Q_{p_{0}} & Q_{p}
\end{array}\right] Z_{k_{0}}^{p_{0}+p}
$$

(2) решение системы (3) единственно тогда и только тогда, когда

$$
\widehat{Y}_{k_{0}} \in \operatorname{colspan}\left[\begin{array}{ll}
Q_{p} & Q_{q}
\end{array}\right] ;
$$

ее единственное решение дается соотношением

$$
\widehat{Y}_{k}=\left[\begin{array}{ll}
Q_{p} & Q_{q}
\end{array}\right] J_{p+q}^{k-k_{0}} Z_{k_{0}}^{p_{0}+p},
$$

где

$$
J_{p+q}^{k-k_{0}}=\left(J_{p}^{-1}\right)^{k-k_{0}} \oplus H_{q}^{k-k_{0}}
$$

u $\widehat{Z}_{k_{0}}^{p+q}$ есть единственное решение алгебраической системь

$$
\widehat{Y}_{k_{0}}=\left[\begin{array}{ll}
Q_{p} & Q_{q}
\end{array}\right] Z_{k_{0}}^{p+q}
$$

(3) решение системы (5) единственно тогда и только тогда, когда

$$
\tilde{Y}_{k_{0}} \in \operatorname{colspan}\left[\begin{array}{ll}
P_{p_{0}}^{T} & P_{p}^{T}
\end{array}\right] ;
$$

ее единственное решение дается соотношением

$$
\tilde{Y}_{k}=\left[\begin{array}{ll}
P_{p_{0}}^{T} & P_{p}^{T}
\end{array}\right]\left(J_{p_{0}+p}^{T}\right)^{k-k_{0}} \widetilde{Z}_{k_{0}}^{p_{0}+p},
$$

где

$$
\left(J_{p_{0}+p}^{T}\right)^{k-k_{0}}=\left(J_{p}^{T}\right)^{k-k_{0}} \oplus H_{q}^{k-k_{0}}
$$

и $\widetilde{Z}_{k_{0}}^{p_{0}+p}$ есть единственное решение алгебраической системь

$$
\tilde{Y}_{k_{0}}=\left[\begin{array}{ll}
P_{p_{0}}^{T} & P_{p}^{T}
\end{array}\right] \widetilde{Z}_{k_{0}}^{p_{0}+p} .
$$


Доказательство. Докажем первое утверждение. Рассмотрим преобразование

$$
Y_{k}=Q Z_{k}
$$

Подставляя вышеприведенное выражение в (1), получаем, что

$$
F Q Z_{k+1}=G Q Z_{k},
$$

и после умножения на $P$ приходим к соотношению

$$
F_{w} Z_{k+1}=G_{w} Z_{k}
$$

Далее, можно записать, что

$$
Z_{k}=\left[\begin{array}{c}
Z_{k}^{p_{0}} \\
Z_{k}^{p} \\
Z_{k}^{q}
\end{array}\right], \quad Z_{k}^{p_{0}} \in \mathcal{M}_{p_{0} n}, \quad Z_{k}^{p} \in \mathcal{M}_{p n}, \quad Z_{k}^{q} \in \mathcal{M}_{p n} .
$$

Учитывая приведенные выше выражения, немедленно получаем три подсистемы (1), именно, подсистемы

$$
\begin{aligned}
Z_{k+1}^{p} & =J_{p_{0}} Z_{k}^{p_{0}}, \\
Z_{k+1}^{p} & =J_{p} Z_{k}^{p}, \\
H_{q} Z_{k+1}^{q} & =Z_{k}^{q} .
\end{aligned}
$$

Подсистемы (27), (28) имеют единственные решения

$$
\begin{aligned}
Z_{k}^{p_{0}}=J_{p_{0}}^{k-k_{0}} Z_{k_{0}}^{p}, & k \geqslant k_{0}, \\
Z_{k}^{p}=J_{p}^{k-k_{0}} Z_{k_{0}}^{p}, & k \geqslant k_{0},
\end{aligned}
$$

соответственно (см. [3, 8, 10, 20]). Для того, чтобы найти решение системы (29), положим $q_{*}$ равным значению индекса нильпотентной матрицы $H_{q}$, то есть

$$
H_{q}^{q_{*}}=0_{q, q} .
$$

Тогда получаем следующие уравнения:

$$
\begin{aligned}
H_{q} Z_{k+1}^{q} & =Z_{k}^{q}, \\
H_{q}^{2} Z_{k+1}^{q} & =H_{q} Z_{k}^{q}, \\
H_{q}^{3} Z_{k+1}^{q} & =H_{q}^{2} Z_{k}^{q}, \\
& \cdots \\
H_{q}^{q^{*}} Z_{k+1}^{q} & =H_{q}^{q^{*}-1} Z_{k}^{q},
\end{aligned}
$$

И

$$
\begin{aligned}
H_{q} Z_{k+1}^{q} & =Z_{k}^{q}, \\
H_{q}^{2} Z_{k+2}^{q} & =H_{q} Z_{k+1}^{q}, \\
H_{q}^{3} Z_{k+3}^{q} & =H_{q}^{2} Z_{k+2}^{q}, \\
& \cdots \\
H_{q}^{q_{*}} Z_{k+q_{*}}^{q} & =H_{q}^{q^{*}-1} Z_{k+q^{*-1}}^{q}
\end{aligned}
$$


Последовательно подставляя каждое уравнение в следующее и используя тот факт, что $H_{q}^{q^{*}}=0_{q, q}$, получаем решение системы (29)

$$
Z_{k}^{q}=0_{q, 1}
$$

Соотношение (16) теперь принимает вид

$$
Q=\left[\begin{array}{lll}
Q_{p_{0}} & Q_{p} & Q_{q}
\end{array}\right]
$$

где $Q_{p_{0}} \in M_{n p_{0}}, Q_{p} \in M_{n p}, Q_{q} \in M_{n q}$ есть матрицы, чьи столбцы образованы, соответственно, $p_{0}, p, q$ обобщенными собственными векторами, соответствующими нулевым, ненулевым конечным и бесконечным собственным значениям. Тогда получаем, что

$$
\begin{aligned}
& Y_{k}=Q Z_{k}=\left[\begin{array}{lll}
Q_{p_{0}} & Q_{p} & Q_{q}
\end{array}\right]\left[\begin{array}{c}
J_{p_{0}}^{k-k_{0}} Z_{k_{0}}^{p_{0}} \\
J_{p}^{k-k_{0}} Z_{k_{0}}^{p} \\
0_{q, 1}
\end{array}\right], \\
& Y_{k}=Q_{p_{0}} J_{p_{0}}^{k-k_{0}} Z_{k_{0}}^{p_{0}}+Q_{p} J_{p}^{k-k_{0}} Z_{k_{0}}^{p},
\end{aligned}
$$

или

$$
Y_{k}=\left[\begin{array}{ll}
Q_{p_{0}} & Q_{p}
\end{array}\right] J_{p_{0}+p}^{k-k_{0}} Z_{k_{0}}^{p_{0}+p}
$$

где

$$
Z_{k_{0}}^{p_{0}+p}=\left[\begin{array}{c}
Z_{k}^{p_{0}} \\
Z_{k}^{p}
\end{array}\right]
$$

Решение существует тогда и только тогда, когда

$$
Y_{k_{0}}=\left[\begin{array}{ll}
Q_{p_{0}} & Q_{p}
\end{array}\right] Z_{k_{0}}^{p_{0}+p}
$$

или $Y_{k_{0}} \in \operatorname{colspan}\left[\begin{array}{ll}Q_{p_{0}} & Q_{p}\end{array}\right]$.

Перейдем к рассмотрению двойственной системы (3). Рассмотрим преобразование

$$
\widehat{Y}_{k}=Q \widehat{Z}_{k}
$$

Подставляя вышеприведенное выражение в (3), получаем, что

$$
F Q \hat{Z}_{k}=G Q \hat{Z}_{k+1},
$$

и после умножения на $P$ приходим к соотношению

$$
F_{w} \widehat{Z}_{k}=G_{w} \widehat{Z}_{k+1} \text {. }
$$

Далее, можно записать, что

$$
\hat{Z}_{k}=\left[\begin{array}{c}
\hat{Z}_{k}^{p_{0}} \\
\widehat{Z}_{k}^{p} \\
\hat{Z}_{k}^{q}
\end{array}\right] .
$$


Учитывая приведенные выше выражения, немедленно получаем три подсистемы (3), именно, подсистемы

$$
\begin{aligned}
J_{p_{0}} \hat{Z}_{k+1}^{p} & =\widehat{Z}_{k}^{p_{0}}, \\
J_{p} \hat{Z}_{k+1}^{p} & =\widehat{Z}_{k}^{p}, \\
\hat{Z}_{k+1}^{q} & =H_{q} \hat{Z}_{k}^{q} .
\end{aligned}
$$

Поскольку

$$
J_{p_{0}}=H_{p_{0}}
$$

система (34) подобна системе (29) и имеет единственное решение

$$
\widehat{Z}_{k}^{p_{0}}=0_{p_{0}, 1}
$$

Жорданова матрица $J_{p}$ невырождена, поскольку она определена для ненулевых конечных собственных значений. Таким образом, подсистемы (35), (36) имеют единственные решения

$$
\begin{array}{ll}
\hat{Z}_{k}^{p}=\left(J_{p}^{-1}\right)^{k-k_{0}} \hat{Z}_{k_{0}}^{p}, & k \geqslant k_{0}, \\
\hat{Z}_{k}^{p}=H_{q}^{k-k_{0}} \widehat{Z}_{k_{0}}^{q}, & k \geqslant k_{0},
\end{array}
$$

соответственно. Тогда, с учетом (16), получаем, что

$$
\begin{aligned}
& \hat{Y}_{k}=Q \widehat{Z}_{k}=\left[\begin{array}{lll}
Q_{p_{0}} & Q_{p} & Q_{q}
\end{array}\right]\left[\begin{array}{c}
0_{p_{0}, 1} \\
\left(J_{p}^{-1}\right)^{k-k_{0}} \widehat{Z}_{k_{0}}^{p} \\
H_{q}^{k-k_{0}} \widehat{Z}_{k_{0}}^{q}
\end{array}\right], \\
& \hat{Y}_{k}=Q_{p}\left(J_{p}^{-1}\right)^{k-k_{0}} \hat{Z}_{k_{0}}^{p}+Q_{q} H_{q}^{k-k_{0}} \widehat{Z}_{k_{0}}^{q},
\end{aligned}
$$

или

$$
\widehat{Y}_{k}=\left[\begin{array}{ll}
Q_{p} & Q_{q}
\end{array}\right] J_{p+q}^{k-k_{0}} \hat{Z}_{k_{0}}^{p+q}
$$

где

$$
\widehat{Z}_{k_{0}}^{p+q}=\left[\begin{array}{c}
\hat{Z}_{k}^{p} \\
\hat{Z}_{k}^{q}
\end{array}\right] .
$$

Решение существует тогда и только тогда, когда

$$
\widehat{Y}_{k_{0}}=\left[\begin{array}{ll}
Q_{p} & Q_{q}
\end{array}\right] \widehat{Z}_{k_{0}}^{p+q}
$$

или $\widehat{Y}_{k_{0}} \in \operatorname{colspan}\left[Q_{p} \quad Q_{q}\right]$.

Перейдем к рассмотрению транспонированной двойственной системы (5). Рассмотрим преобразование

$$
\tilde{Y}_{k}=P^{T} \tilde{Z}_{k}
$$


Подставляя вышеприведенное выражение в (5), получаем, что

$$
F^{T} P^{T} \widetilde{Z}_{k+1}=G^{T} P^{T} \widetilde{Z}_{k}
$$

или

$$
\widetilde{Z}_{k+1}^{T} P F=\widetilde{Z}_{k}^{T} P G,
$$

и после умножения на $Q$ приходим к соотношениям

$$
\widetilde{Z}_{k+1}^{T} F_{w}=\widetilde{Z}_{k}^{T} v G_{w}
$$

или

$$
F_{w}^{T} \widetilde{Z}_{k+1}=G_{w}^{T} \tilde{Z}_{k}
$$

Производя замену

$$
\widetilde{Z}_{k}=\left[\begin{array}{c}
\widetilde{Z}_{k}^{p_{0}} \\
\widetilde{Z}_{k}^{p} \\
\widetilde{Z}_{k}^{q}
\end{array}\right],
$$

немедленно приходим к трем подсистемам (5), именно, подсистемам

$$
\begin{aligned}
\widetilde{Z}_{k+1}^{p} & =J_{p_{0}}^{T} \widetilde{Z}_{k}^{p_{0}}, \\
\widetilde{Z}_{k+1}^{p} & =J_{p}^{T} \widetilde{Z}_{k}^{p}, \\
H_{q}^{T} \widetilde{Z}_{k+1}^{q} & =\widetilde{Z}_{k}^{q} .
\end{aligned}
$$

Система (43) подобна системе (29) и имеет единственное решение

$$
\widetilde{Z}_{k}^{p_{0}}=0_{p_{0}, 1}
$$

Системы (41), (42) имеют единственные решения

$$
\begin{array}{ll}
\widetilde{Z}_{k}^{p}=\left(J_{p_{0}}^{T}\right)^{k-k_{0}} \widetilde{Z}_{k_{0}}^{p_{0}}, \quad & k \geqslant k_{0}, \\
\widetilde{Z}_{k}^{p}=\left(J_{p}^{T}\right)^{k-k_{0}} \widetilde{Z}_{k_{0}}^{p}, & k \geqslant k_{0},
\end{array}
$$

соответственно. Из (15) получаем, что

$$
P^{T}=\left[\begin{array}{ccc}
P_{p_{0}}^{T} & P_{p}^{T} & P_{q}^{T}
\end{array}\right], \quad P_{p_{0}}^{T} \in \mathcal{M}_{n p_{0}}, \quad P_{p}^{T} \in \mathcal{M}_{n p}, \quad P_{q}^{T} \in \mathcal{M}_{n q},
$$

откуда получаем, что

$$
\begin{aligned}
& \tilde{Y}_{k}=P^{T} \widetilde{Z}_{k}=\left[\begin{array}{lll}
P_{p_{0}}^{T} & P_{p}^{T} & P_{q}^{T}
\end{array}\right]\left[\begin{array}{c}
\left(J_{p_{0}}^{T}\right)^{k-k_{0}} \\
\left(J_{p}^{T}\right)^{k-k_{0}} \widetilde{Z}_{k_{0}}^{p} \\
0_{q, 1}
\end{array}\right], \\
& \tilde{Y}_{k}=P_{p_{0}}^{T}\left(J_{p_{0}}^{T}\right)^{k-k_{0}} \widetilde{Z}_{k_{0}}^{p_{0}}+P_{p}^{T}\left(J_{p}^{T}\right)^{k-k_{0}} \widetilde{Z}_{k_{0}}^{p},
\end{aligned}
$$

или

$$
\tilde{Y}_{k}=\left[\begin{array}{ll}
P_{p_{0}}^{T} & P_{p}^{T}
\end{array}\right]\left(J_{p_{0}+q}^{T}\right)^{k-k_{0}} \widetilde{Z}_{k_{0}}^{p_{0}+p},
$$


где

$$
\widetilde{Z}_{k_{0}}^{p_{0}+p}=\left[\begin{array}{c}
\widetilde{Z}_{k}^{p_{0}} \\
\widetilde{Z}_{k}^{p}
\end{array}\right]
$$

Решение существует тогда и только тогда, когда $\tilde{Y}_{k_{0}} \in \operatorname{colspan}\left[\begin{array}{cc}P_{p_{0}}^{T} & P_{p}^{T}\end{array}\right]$.

Замечание 2. Пусть $\tilde{q}-$ индекс матрицы $H_{q}$, тогда для $k \geqslant k_{0}+\tilde{q}$ мы видим, что

$$
H_{q}^{k-k_{0}}=0,
$$

и аналитическое решение (24) имеет вид

$$
\tilde{Y}_{k}=Q_{p}\left(J_{p}^{-1}\right)^{k-k_{0}} \widetilde{Z}_{k_{0}}^{p} .
$$

Поэтому решение (24) можно также записать в виде

$$
\tilde{Y}_{k}=Q_{p}\left(J_{p}^{-1}\right)^{k-k_{0}} \widetilde{Z}_{k_{0}}^{p}+Q_{q} \sum_{i=0}^{\tilde{q}-1} \delta_{k-k_{0}-i} H_{q}^{i} \widetilde{Z}_{k_{0}}^{q}
$$

где $\delta_{k-a}$ есть функция Дирака

$$
\delta_{k-a}= \begin{cases}1, & k=a \\ 0, & k \neq a\end{cases}
$$

\section{4. Численные примеры}

Рассмотрим матричный пучок $s F-G$ и положим

$$
F=\left[\begin{array}{rrrrr}
1 & 0 & -1 & -1 & 1 \\
0 & -2 & 0 & 1 & 1 \\
-2 & -2 & 2 & 2 & 0 \\
0 & 2 & 1 & 0 & -2 \\
0 & 0 & 0 & 0 & 0
\end{array}\right], \quad G=\left[\begin{array}{rrrrr}
-1 & 0 & 1 & 1 & 0 \\
-4 & -2 & 5 & 5 & -2 \\
-2 & -2 & 2 & 2 & 0 \\
4 & 2 & -4 & -4 & 1 \\
-1 & 0 & 2 & 1 & -1
\end{array}\right]
$$

Тогда

$$
\operatorname{det}[s F-G]=(s-2)(s-1) .
$$

Конечные элементарные делители есть $s-2, s-1$, и $\hat{s}^{3}$ есть бесконечные элементарные делители. Найдутся невырожденные матрицы

$$
P=\left[\begin{array}{rrrrr}
0 & 1 & 0 & 1 & 0 \\
0 & 0 & -1 & 0 & 0 \\
1 & 0 & 0 & 0 & -1 \\
1 & 0 & 1 & 1 & 0 \\
0 & 0 & 0 & 0 & 1
\end{array}\right], \quad Q=\left[\begin{array}{lllll}
1 & 0 & 0 & 1 & 0 \\
0 & 1 & 1 & 0 & 1 \\
0 & 0 & 0 & 1 & 1 \\
1 & 0 & 1 & 0 & 0 \\
0 & 0 & 1 & 1 & 1
\end{array}\right]
$$

такие, что

$$
P F Q=F_{w}=\left[\begin{array}{cc}
I_{p} & 0_{2,3} \\
0_{3,2} & H_{q}
\end{array}\right], \quad G_{w}=\left[\begin{array}{cc}
J_{p} & 0_{2,3} \\
0_{3,2} & I_{q}
\end{array}\right],
$$


где

$$
J_{p}=\left[\begin{array}{ll}
1 & 0 \\
0 & 2
\end{array}\right], \quad J_{p}^{k}=\left[\begin{array}{cc}
1 & 0 \\
0 & 2^{k}
\end{array}\right], \quad H_{q}=\left[\begin{array}{lll}
0 & 1 & 0 \\
0 & 0 & 1 \\
0 & 0 & 0
\end{array}\right]
$$

Далее,

$$
Q_{p}=\left[\begin{array}{ll}
1 & 0 \\
0 & 1 \\
0 & 0 \\
1 & 0 \\
0 & 0
\end{array}\right], \quad Q_{q}=\left[\begin{array}{lll}
0 & 1 & 0 \\
1 & 0 & 1 \\
0 & 1 & 1 \\
1 & 0 & 0 \\
1 & 1 & 1
\end{array}\right], \quad P_{p}^{T}=\left[\begin{array}{rr}
0 & 0 \\
1 & 0 \\
0 & -1 \\
1 & 0 \\
0 & 0
\end{array}\right]
$$

Поскольку пучок не имеет нулевых собственных значений, матрицы $Q_{p_{0}}, P_{p_{0}}$ не существуют.

Пример 1. Рассмотрим систему (1), и пусть начальные условия есть

$$
Y_{0}=\left[\begin{array}{l}
1 \\
2 \\
0 \\
1 \\
0
\end{array}\right]
$$

Then $Y_{0} \in \operatorname{colspan} Q_{p}$, и по теореме 1 система имеет единственное решение, задаваемое соотношением

$$
Y_{k}=\left[\begin{array}{cc}
1 & 0 \\
0 & 2^{k} \\
0 & 0 \\
1 & 0 \\
0 & 0
\end{array}\right] Z_{0}^{p}
$$

вычисляя $Z_{0}^{p}$, получаем

$$
Y_{0}=\left[\begin{array}{ll}
1 & 0 \\
0 & 1 \\
0 & 0 \\
1 & 0 \\
0 & 0
\end{array}\right] Z_{0}^{2}
$$

или

$$
Z_{0}^{p}=\left[\begin{array}{l}
1 \\
2
\end{array}\right]
$$

таким образом, решение системы есть

$$
Y_{k}=\left[\begin{array}{c}
1 \\
2^{k+1} \\
0 \\
1 \\
0
\end{array}\right] .
$$


Теперь рассмотрим систему (1) с начальным условием

$$
Y_{0}=\left[\begin{array}{l}
0 \\
0 \\
0 \\
1 \\
1
\end{array}\right]
$$

Тогда $Y_{0} \notin \operatorname{colspan} Q_{p}$, и из теоремы 1 следует, что система имеет не единственное решение. Ее бесконечно много решений даются соотношением

$$
Y_{k}=\left[\begin{array}{cc}
1 & 0 \\
0 & 2^{k} \\
0 & 0 \\
1 & 0 \\
0 & 0
\end{array}\right] C
$$

где

$$
C=\left[\begin{array}{l}
c_{1} \\
c_{2}
\end{array}\right]
$$

есть константа и размерность пространства векторов решений системы равна двум.

Пример 2. Рассмотрим систему (3). Так как матрицы $Q_{p_{0}}$ не существует,

$$
Q=\operatorname{colspan}\left[Q_{p} \quad Q_{q}\right] \text {. }
$$

Следовательно, $Y_{0} \in \operatorname{colspan}\left[Q_{p} \quad Q_{q}\right]$, для любого начального условия (5) системы, и система всегда имеет единственное решения. Например, положим начальное условие для системы (3) равным

$$
Y_{0}=\left[\begin{array}{l}
0 \\
0 \\
0 \\
1 \\
1
\end{array}\right]
$$

Индекс $H_{q}$ равен $\tilde{q}=3$, и из (47) следует, что единственное решение системы есть

$$
\widehat{Y}_{k}=Q_{p}\left(J_{p}^{-1}\right)^{k} \hat{Z}_{0}^{p}+Q_{q} \sum_{i=0}^{2} \delta_{k-i} H_{q}^{i} \widehat{Z}_{0}^{q},
$$

или

$$
\widehat{Y}_{k}=\left[\begin{array}{cc}
1 & 0 \\
0 & (1 / 2)^{k} \\
0 & 0 \\
1 & 0 \\
0 & 0
\end{array}\right] \widehat{Z}_{0}^{p}+Q_{q} \sum_{i=0}^{2} \delta_{k-i} H_{q}^{i} \widehat{Z}_{0}^{q}
$$


вычисляя $\hat{Z}_{0}^{p}$ и $\hat{Z}_{0}^{q}$, получаем, что

$$
Y_{0}=\left[\begin{array}{ll}
1 & 0 \\
0 & 1 \\
0 & 0 \\
1 & 0 \\
0 & 0
\end{array}\right] \hat{Z}_{0}^{p}+Q_{q} \hat{Z}_{0}^{q}
$$

или

$$
\hat{Z}_{0}^{p}=\left[\begin{array}{r}
0 \\
-1
\end{array}\right], \quad \hat{Z}_{0}^{q}=\left[\begin{array}{l}
1 \\
0 \\
0
\end{array}\right]
$$

и решение системы есть

$$
\widehat{Y}_{k}=\left[\begin{array}{c}
0 \\
(1 / 2)^{k}+\delta_{k} \\
0 \\
\delta_{k} \\
\delta_{k}
\end{array}\right]
$$

Пример 3. Рассмотрим систему (5), и положим начальное условие системы равным

$$
\tilde{Y}_{0}=\left[\begin{array}{l}
0 \\
1 \\
1 \\
1 \\
0
\end{array}\right]
$$

Тогда $\tilde{Y}_{0} \in \operatorname{colspan} P_{p}^{T}$, и единственное решение системы задается соотношением

$$
\tilde{Y}_{k}=\left[\begin{array}{cc}
0 & 0 \\
1 & 0 \\
0 & -2^{k} \\
1 & 0 \\
0 & 0
\end{array}\right] \widetilde{Z}_{0}^{p}
$$

вычисляя $\widetilde{Z}_{0}^{p}$, получаем, что

$$
\tilde{Y}_{0}=\left[\begin{array}{rr}
0 & 0 \\
1 & 0 \\
0 & -1 \\
1 & 0 \\
0 & 0
\end{array}\right] \widetilde{Z}_{0}^{p},
$$

или

$$
\widetilde{Z}_{0}^{p}=\left[\begin{array}{r}
1 \\
-1
\end{array}\right]
$$


и тогда решение системы равно

$$
Y_{k}=\left[\begin{array}{c}
0 \\
1 \\
2^{k} \\
1 \\
0
\end{array}\right]
$$

Далее, положим начальное условие равным

$$
Y_{0}=\left[\begin{array}{l}
0 \\
0 \\
0 \\
1 \\
1
\end{array}\right]
$$

Тогда $Y_{0} \notin$ colspan $P_{p}^{T}$, и система имеет бесконечно много решений

$$
Y_{k}=\left[\begin{array}{cc}
0 & 0 \\
1 & 0 \\
0 & -2^{k} \\
1 & 0 \\
0 & 0
\end{array}\right] C,
$$

где

$$
C=\left[\begin{array}{l}
c_{1} \\
c_{2}
\end{array}\right]
$$

- константа и размерность пространства векторов решений равна двум.

\section{5. Заключение}

Целью статьи являлось установление связи между решениями систем, двойственных к данной обобщенной автономной системе с дискретным временем вида (1). Мы установили, что если каноническая форма Вейерштрасса, равно как матрицы преобразования $P$ и $Q$, являются известными для пучка $s F-G$, то возможно предъявить необходимые и достаточные условия существования и единственности решений систем вида (3) и (5). Это позволяет получить формулы для решения каждой из этих систем в случае их существования.

Автор выражает искреннюю благодарность проф. Г. И. Калогеропулосу за плодотворные обсуждения, которые способствовали улучшению статьи.

\section{Список литературы}

1. Campbell S. L., Singular systems of differential equations, 1-2. Pitman, San Francisco, 1980, 1982.

2. Sandefur J. T., Discrete dynamical systems. Theory and applications. Clarendon Press, Oxford, 1990.

3. Steward G. W., Sun J. G., Matrix perturbation theory. Oxford University Press, Oxford, 1990.

4. Kalogeropoulos G. I., Matrix pencils and linear systems, PhD Thesis. City University, London, 1985. 
5. Vizireanu D. N., A fast, simple and accurate time-varying frequency estimation method for singlephase electric power systems. Measurement (2012) 45, №5, 1331-1333.

6. Vizireanu D. N., Halunga S. V., Simple, fast and accurate eight points amplitude estimation method of sinusoidal signals for DSP based instrumentation. J. Instrumentation (2012) 7, №4, P04001.

7. Dai L., Singular control systems. Springer, Berlin, 1989.

8. Гантмахер Ф. Р., Теория матрии. Наука, Москва, 1967.

9. Gohberg I., Lancaster P., Rodman L., Matrix polynomials. Academic Press, New York, 1983.

10. Klamka J., Controllability of dynamical systems. Matematyka Stosowana (2008) 50, №9, 57-75.

11. Klamka J., Controllability of nonlinear discrete systems. Int. J. Appl. Math. Comput. Sci. (2002) 12, №2, 173-180.

12. Klamka J., Controllability of dynamical systems. Kluwer, Dordrecht, 1991.

13. Dassios I. K., Solutions of higher-order homogeneous linear matrix differential equations for consistent and non-consistent initial conditions: regular case. ISRN Mathematical Analysis (2011) 2011, 183795 .

14. Dassios I. K., On a boundary value problem of a class of generalized linear discrete-time systems. Advances in Difference Equations (2011) 2011, 51.

15. Dassios I. K., Perturbation and robust stability of autonomous singular linear matrix difference equations. Appl. Math. Comput. (2012) 218, 6912-6920.

16. Mitrouli M., Kalogeropoulos G., Generalised linear discrete-time systems and matrix pencils algebraic duality. J. Inst. Math. Comput. Sci., Math. Ser. (1997) 10, №2, 81-90.

17. Mitrouli M., Kalogeropoulos G., A matrix pencil approach computing the elementary divisors of a matrix: Numerical aspects and applications. Korean J. Comput. Appl. Math. (1998) 5, №3, 627-644.

18. Mitrouli M., Kalogeropoulos G., A compound matrix algorithm for the computation of the Smith form of a polynomial matrix. Numer. Algorithms (1994) 7, 145-159.

19. Lewis F. L., A survey of linear singular systems. Circuits Syst. Signal Process. (1986) 5, 3-36.

20. Rugh W. J., Linear system theory. Prentice Hall, London, 1996.

Статья поступила 8.06.2011. Переработанный вариант поступил 1.11.2012. 